\title{
EL ORDEN DE PALABRAS DEL ALEMÁN Y SU ADQUISICIÓN
}

\author{
Christoph Ehlers
}

\begin{abstract}
German word order and its acquisition is an appealing subject of study for generativism, since the visibility of verbal elements allows formal description and explanation. The first part of this article gives a short overview of the question of basic word order/d-structure of German, which is commonly described as head-final. In the second part we deal with three different GBanchored models of acquisition of word order of German as a second language, with special emphasis on the question of L1 influence.
\end{abstract}

Las particularidades del orden de palabras (OP) alemán han sido y siguen siendo un reto tanto para los que lo investigan como para los que lo quieran aprender. Para aquellos lectores que no hayan intentado todavía ninguna de las dos cosas, valgan como advertencia las siguientes observaciones de Mark Twain, extraídas de su glosa "La terrible lengua alemana":

“... Los libros alemanes son bastante fáciles de leer con la ayuda de un espejo o si uno se pone a hacer el pino para darle el orden correcto a la construcción, mas comprender un periódico alemán, al extranjero nunca le será posible...En los periódicos alemanes el verbo siempre aparece en la página siguiente, e incluso he oído que en ocasiones los periodistas tienen que terminar con prisa después de haberse explayado durante algunas columnas en intrigantes preludios y paréntesis [sintácticos] hasta que finalmente tienen que pasar a imprenta sin haber llegado siquiera al verbo, lo cual por supuesto hace que el lector termine exhausto y tan ignorante como antes...". "Tienen los alemanes también una especie de paréntesis que forman partiendo el verbo en dos y poniendo una mitad al principio y la otra al final. ¿Puede imaginarse algo más confuso? Estos engendros se llaman 'verbos separables’, y la gramática alemana está cubierta de ellos, igual que las pústulas de un herpes..."

Si el bueno de Twain todavía viviera éste sería un buen momento para intentar calmarlo y explicarle las regularidades que se esconden detrás de toda esta confusión aparente. ¿Y qué mejor manera de hacerlo que echando mano del modelo gramatical creado por su paisano Noam Chomsky, cuyo objetivo último es precisamente eso: explicar por qué las lenguas son como son.

Para ello, este trabajo se estructurará en dos partes: primero, se comentarán las distintas propuestas generativistas acerca del OP del alemán, con especial atención a la cuestión del orden básico o subyacente, y, segundo, se realizará un recorrido por el estado actual de la investigación en cuanto a la adquisición del OP alemán como segunda lengua, en el que nos 
centraremos en el papel de la transferencia (TF) y su reinterpretación total en el contexto de la Gramática Universal chomskiana.

De entrada, conviene tener presente que en el paradigma chomskiano, ya desde Aspects (1965) pero sobre todo desde el modelo de Principios y Parámetros (Chomsky 1982) se establece una estrecha relación entre la forma de la gramática y su adquisición, también como segunda lengua. La observación del proceso de maduración en el que se súceden en el aprendiente los distintos sistemas transicionales o interlenguas (IL) suministra valiosas evidencias acerca de la existencia y el funcionamiento de la facultad del lenguaje o Gramática Universal.

Veamos, pues, en primer lugar, las características secuenciales más importantes del alemán.

\section{EL ORDEN DE PALABRAS DEL ALEMÁN}

La característica más general de la serialización dentro de la oración alemana es el comportamiento completamente distinto de sus constituyentes nominales y verbales. Mientras los primeros poseen una considerable libertad de movimiento o scrambling, relacionada con la riqueza de su sistema morfológico, los elementos verbales están firmemente anclados en una serie de posiciones gramaticalizadas. A diferencia del $45 \%$ de las lenguas del mundo, y entre ellas el español, que presentan un orden no marcado SVO (Siewierska 1994), el alemán posee, a primera vista, nada menos que tres órdenes posibles, todos ellos obligatorios en determinados contextos. El verbo finito debe aparecer en primer, segundo o último lugar de la oración, dependiendo del tipo de ésta, y el no-finito siempre en posición final y desgajado de su auxiliar o modal.

V-fin: (1) Ich glaube, dass Otto sich einen Helm gekauft hat.

('Creo que Otto se un casco comprado ha').

V-2: (2) Gestern hat Otto sich einen Helm gekauft.

('Ayer ha Otto se un casco comprado')

V-1: (3) Hat Otto sich wirklich einen Helm gekauft?

( ¿Ha Otto se realmente un casco comprado?’)

Son destacables sobre todo tres características:

- V-final en oración subordinada introducida por complementante

- V-finito y complementante están en distribución complementaria. 
- V-2 en oraciones no incrustadas, con el efecto de la inversión entre verbo y sujeto cuando la oración comienza por otro constituyente que no sea el sujeto. (2).

Esta heterogeneidad sintáctica supone a la vez un reto y una oportunidad para una teoría formal del lenguaje como el generativismo. Un reto porque una teoría gramatical que reclama para sí poseer adecuación explicativa tiene que reducir una serie de estructuras derivadas en la estructura-s a una sola subyacente. ¿Cómo afrontaría este modelo gramatical el hecho de que las regularidades topológicas de la sintaxis compleja del alemán parecen obligar a suponer diferentes estructuras oracionales según la posición del verbo? Anticipemos una primera solución: suponiendo que entre los distintos tipos de posición verbal debe haber relaciones estructurales sistemáticas, estas correlaciones habrían de representarse de manera transformacional.

La oportunidad, por el otro lado, consiste en el hecho de que la topología de los constituyentes verbales no está sometida, a diferencia de los elementos nominales, a restricciones funcionales de tipo informativo sino que se encuentra regulada por estrictos criterios formales. Ofrece un asidero seguro y bien visible en un "terreno tan resbaladizo" (Hernanz y Brucart 1987:79) como el del orden, permitiendo excluir de antemano y nítidamente cualquier tipo de explicación semántica o pragmática. De este modo se justifica el gran atractivo que este fenómeno ejerce en aquellos estudiosos de los procesos de adquisición L1 y L2 que quieran dar cuenta del módulo cognitivo responsable del procesamiento, la producción y la adquisición de las estructuras formales, la core grammar de una lengua.

\section{EL ORDEN BÁSICO O SUBYACENTE: ¿SVO O SOV?}

De entrada, entre las posibilidades VO y OV existe una larga controversia tipológica

"con tendencia a manifestarse ideológicamente: los tipologistas de estructura superficial tienden a preferir el orden SVO de la oración principal como menos marcado, mientras que los gramáticos generativistas tienden a considerar más básico el orden de palabras de la oración subordinada" (Comrie 1988:132).

Pero también dentro del propio marco generativista se ofrecen, como no, distintos análisis en función de las asunciones teóricas subyacentes. Aquí, como tendremos ocasión de ver, se oponen principalmente las versiones más estándar de la teoría de Rección y Ligamento (Chomsky 1981) y su modelo de Principios y Parámetros, con las propuestas más recientes de Kayne (1993), Zwart (1993) asumidas en parte en el Programa Minimalista de Chomsky (1995). Los primeros se decantan preferentemente por SOV generado en la base, los segundos por un orden universal SVO.

En efecto, desde los trabajos fundacionales que aplican la gramática generativa al alemán - Bach (1962), Bierwisch (1963), y Thiersch (1978) en su tesis doctoral dirigida por el propio Chomsky) - , el análisis generativista se decanta claramente por un orden básico SOV: "There seems to be a general consensus now that the underlying position of the verb is at the end of the verb phrase" (Clahsen y Muysken 1986:68). 
Enumeremos los principales argumentos que han llevado a este "consenso general":

- En primer lugar, una razón metodológica de la teoría estándar: la suposición de V-final generado en la base confiere coherencia al análisis formal, pues en la teoría estándar, el orden se determina sobre el parámetro del núcleo. Dicho de manera muy escueta, las lenguas son fundamentalmente de núcleo inicial (inglés) o de núcleo final (japonés, turco, alemán), parametrizando en cada sentido la estructura sintagmática universal recogida en la teoría X-barra. Los núcleos ejercen la rección léxica $\mathrm{y}$, como tales, pueden asignar roles temáticos (agente, receptor, paciente etc.). en una de dos direcciones. La dirección de la asignación de los papeles temáticos, hacia la derecha o la izquierda, es la base gramatical del orden de palabras en el marco de la rección léxica ${ }^{1}$ De este modo, la suposición SOV permite un tratamiento sintáctico más acorde con la caracterización de las lenguas germánicas-occidentales como head-final en todo tipo de sintagmas"

- Otro argumento a favor de la posición final del verbo proviene de los hechos observados durante la adquisición: el niño que adquiere el alemán muestra una fuerte preferencia por las construcciones V-final. Clahsen y Muysken (1986) observan una frecuencia del 60 al $70 \%$ de estructuras Vfinal en el lenguaje infantil; Poeppel y Wexler (1993) e Ingram y Thompson (1996) establecen porcentajes parecidos.

- Otra evidencia más es la colocación final del núcleo en las construcciones de numerales, de infinitivo y de imperativo (Bierwisch 1964:35): en el sistema numérico, unidades y decenas se invierten ("cincuenta y tres" es tres y cincuenta), la forma de cita de los verbos y sus argumentos siempre es con el infinitivo al final (jemandem etwas geben, "a alguien algo dar") y la misma linearización modificador - modificado opera en un tipo de imperativos (Bitte die Tür schließen, "Por favor la puerta cerrar").

Con respecto al orden de palabras, conviene recordar que para la teoría estándar los principios de la teoría de la $\mathrm{X}^{\prime}$ se ocupan exclusivamente de las relaciones universales de dominio y rección, mientras que la serialización o las relaciones secuenciales, específicas de cada lengua, son determinadas por los parámetros. Para el caso del alemán, el movimiento verbal implicado en estas regularidades es producto de dos fuerzas: por un lado, las restricciones que afectan la interacción entre la estructura universal de la oración y las posibles realizaciones parametrizadas de las proyecciones funcionales COMP y FLEX,

\footnotetext{
${ }^{1}$ No obstante, algunas lenguas parecen ser bidireccionales (cfr. Hoekstra 1984). Por motivos de economía de reglas, se considera como no-marcada la rección unidireccional y marcada la bidireccional.

2 El argumento "clásico" (p.e. Greenberg 1963) se basa en el hecho tipológico de que el alemán y otras lenguas germánico-occidentales posean postposiciones, una clase de palabras normalmente reservada a las lenguas de orden SOV En el vasco se da la misma implicación entre el orden OV y la postposición.
} 
específicas en cada lengua, así como, por el otro, las particularidades del conjunto de movimientos denominado estrategia V-2. (Travis 1991).

Tres son los principios fundamentales que restringen el movimiento verbal ${ }^{3}$ : Principio de Proyección ('cualquier movimiento deja una huella'), Principio de Subyacencia ('ningun constituyente puede atravesar más de un nudo límite') y Principio de la Categoría Vacía ('una huella debe estar propiamente regida').

Veamos la propuesta de Travis (1991) que nos puede servir de referencia en un mundillo científico "orfebreril" (Demonte 1989:25) como el generativista donde las teorías y propuestas nacen y mueren a ritmo de correo electrónico. Fundamentalmente, Travis combina los efectos de los parámetros, las restricciones sobre mueve- $\alpha$ y el Principio de la Categoría Vacía $(P C V) .{ }^{4}$ Como punto de partida, recoge el consenso general que el primer parámetro responsable de la secuenciación es el de núcleo-final/inicial: los sintagmas verbales del inglés, del islandés y del yiddish son de núcleo inicial, mientras en sueco y alemán son de núcleo final. La colocación V-2 del verbo flexionado es consecuencia de un movimiento núcleo a núcleo. El proceso de transformación desde la estructura profunda hacia la superficial según Travis (1991) sería el siguiente:

\section{Estrategia V-2:}

\section{Parámetro 1: Núcleo final/inicial}

Estructura-P:

(1) *[FLex Otto einen Helm gekauft [hat $]]]$

'Otto un casco comprado ha.'

Estando FLEX $^{\circ}$ vacío y adecuadamente gobernado por su Especificador, hat será obligado a salir de su posición final, generado en la base, hacía esta posición mediante un movimiento núcleo a núcleo. El resultado en la estructura-S es el siguiente:

(2) [FleX, Otto[FLeX hat [sv einen Helm gekauft (vacío)]]]

'Otto ha un casco comprado'

La categoría vacía en $\mathrm{V}$ será identificada puesto que está adecuadamente gobernada al ser el núcleo del complemento de FLEX, y recibirá sus rasgos del nudo FLEX que la domina adecuadamente.

\footnotetext{
${ }^{3}$ Se observará que aquí se aplican en el interior de la oración, no en extracciones de largo alcance como "¿Quién me dijiste que te contó que se casaba?"

${ }^{4}$ (Lasnik y Saito 1984) Regula las condiciones en las que son reconocibles las huellas del movimiento. Chomsky (1981): "Una categoría vacía debe estar regida propiamente" Una categoría léxica propiamente regida puede desplazarse todo lo lejos que se quiera ya que la rección de su huella se mantendrá constante.
} 
Ahora bien, cuando una categoría que no sea el sujeto encabeza la oración ("heute"), ésta aparecerá en la posición ESP(ecificador) de COMP.

Estructura-P:

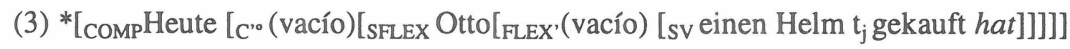

'Hoy Otto un casco comprado ha'.

Puesto que ni la categoría vacía en COMP ni la de FLEX están identificadas, el núcleo haben es obligado a moverse a través de FLEX hacía COMP:

Estructura-S:

(4) [comp heute [c' hat[SFLeX Otto[r' (vacío) [sv einen Helm gekauft]]]]]

'Hoy ha Otto un casco comprado'

\section{No V-2 en otras lenguas}

\section{Parámetro 2: Adjunción a FLEX posible/imposible}

La misma oración en inglés es generada mediante adjunción ${ }^{5}$ de "today" a FLEX, lo cual no es posible en alemán, pero sí en español (Torrego 1984):

(5) *[SFlex Heute [FleX' die Kinder [sv haben das Brot gegessen]

'Hoy los niños han el pan comido'

Ya que "hoy" no aparece en la posición ESP de COMP, no hay ningún COMP vacío que tuviera que llenarse mediante el movimiento del verbo para evitar la violación del Principio de la Categoría Vacía.

\section{Distribución complementaria de movimiento verbal y COMP}

En la representación en estructura-S

(6) Ich glaube, [scompdass [SFLEX Otto [FLeX' (vacío)[sv einen Helm t $\mathrm{j}_{\mathrm{j}}$ gekauft hat]]]]

PRO creo que Otto un casco comprado ha

Travis (1984) explica el hecho de que el verbo permanezca en su posición final generada en la base en vez de moverse para llenar la categoría FLEX con otro parámetro:

\footnotetext{
${ }^{5}$ Adjunción "En las estructuras de adjunción, un $\mathrm{X}_{\mathrm{i}}$ cualquiera (núcleo, complemento o especificador) domina a otro $\mathrm{X}_{\mathrm{i}}$ ' y se supone que los constituyentes así configurados tienen el mismo rango sintáctico y semántico" (Demonte 1989:46) Ejemplos: por entre las ramas, tan verdaderamente digno de ser visto.
} 


\section{Parámetro 3: COMP puede identificar rasgos de FLEX}

En el marco de la teoría de Rección y Ligamento, una categoría sólo puede quedarse vacía si es regida apropiadamente. Pero en alemán y sueco, a diferencia del inglés y del islandés, el complementante léxico COMP "dass" lleva rasgos que le capacitan para identificar y regir apropiadamente el núcleo vacío de su complemento FLEX. Además, cuando un hueco ha sido llenado con su conjunto de rasgos mediante la rección apropiada, aunque permanezca léxicamente vacío, no puede servir como "lugar de aterrizaje". Esta circunstancia también podría explicar el "preposition stranding" en las oraciones incrustadas del inglés.

Merece la pena detenerse un poco en la distribución complementaria entre COMP léxico y V-finito que en todo caso radica en la fuerte relación entre COMP y FLEX. Ya Platzack (1983) y Haider (1986) en su "Hipótesis de Adjunción" plantearon la hipótesis de que el alemán, y otras lenguas germánico-occidentales, no poseen un nudo FLEX independiente y separado de COMP sino que los funden en uno, que denominan CONFL. Afirman que el parámetro COMP-FLEX, que altera la posición y función de los nudos COMP y FLEX, es el responsable de una gran variedad de hechos observables en el orden de palabras de las lenguas germánicas.

Así, en la estructura-p, FLEX se encuentra en COMP. Lo avalan los datos contrastivos: a diferencia del inglés, no representa una posición independiente, lo cual explica que el alemán, el sueco y el islandés manifiestan los efectos de V-2, mientras que en inglés no existen. En todas aquellas lenguas, el efecto V-2 desaparece, como hemos visto más arriba, con la aparición de un complementante léxico, como "dass" en alemán, que estando en distribución complementaria con el verbo, hace que éste permanezca en su posición final. La fusión de COMP y FLEX en un único nudo puede justificar este hecho: al estar COMP ya ocupado por el elemento léxico "dass", el verbo no puede moverse hacia su posición FLEX.

Este hecho es observable en un fenómeno del bávaro, tildado en el alto alemán como craso vulgarismo o aún error ${ }^{6}$ : la flexión de la conjunción o del sintagma-QU:

I woass net, obst a Spitzbua bist

('No sé si-(FLEX 2a Sg.) un bribón ser-(FLEX 2 ${ }^{\mathrm{a}} \mathrm{Sg}$.)')

De acuerdo con Travis (1991) serían, pues, estos tres parámetros (núcleo final/inicial, adjunción a FLEX posible/imposible, COMP identifica los rasgos de FLEX) los responsables de las particularidades del orden de palabras del alemán. Su principal ventaja consiste en combinar el poder explicativo del PCV con los otros parámetros ya establecidos.

\footnotetext{
${ }^{6}$ Algo parecido al interrogativo español “¿cuála?”.
} 
Todo ello presupone SOV. Pero volviendo a la cuestión del orden subyacente VO/OV otros autores generativistas pronto presentaron un modelo alternativo, según el cual el orden SVO no es producto de las transformaciones sino que es generado directamente en la base. Para ellos, sería poco plausible suponer que las oraciones subordinadas (V-final) son las más fáciles de generar. Las oraciones más simples y menos marcadas presentan siempre el orden SVO.

A la misma conclusión, aunque por otros caminos, llegan las alternativas radicales más recientes de Kayne (1994) y Zwart (1991) asumidas en parte por Chomsky (1995) en la teoría minimalista: la secuenciación obedece universalmente a una jerarquía establecida por el Axioma de Correpondencia Lineal según el cual el mando-c asimétrico determina la secuenciación de los elementos terminales. De este modo,. hay un orden universal Esp-Xcomp (SVO) y los casos en los que este orden se invierte (SOV), serían derivados por un movimiento parametrizado del objeto a través del verbo, al contrario que en las propuestas anteriores, donde para el alemán se parametriza SOV en la base y se deriva SVO en la estructura-s. A diferencia del análisis anterior que supone un SV de núcleo final para el alemán y de núcleo inicial para el inglés, un análisis en la línea de Kayne (1993) requeriría que el objeto ascendiera a la posición de Especificador de alguna proyección funcional por encima de SV mientras que el objeto inglés (y español) permanece en su lugar en el SV.

Desde este punto de vista, se suele explicar el bloqueo del movimiento V2 en oraciones subordinadas (distribución complementaria entre conjunción y verbo en ors. subords.) localizando el "rasgo de fortaleza", que desencadena el movimiento del verbo hacía la izquierda, bajo COMP. Situando aquí el rasgo fuerza, el complementante léxico, situado en COMP, puede cotejar y bloquearlo en el componente fonológico.

Situemos ahora estas discusiones en el contexto de la investigación de la adquisición de segundas lenguas.

\section{LA GRAMÁTICA UNIVERSAL EN LA ADQUISICIÓN DE SEGUNDAS LENGUAS}

El rasgo que quizás mejor caracterice la adquisición de una segunda lengua frente a la de la primera es la heterogeneidad de sus resultados. Las variables responsables de esta diversidad son de tipo externo (entorno formal o natural, metodología, factores sociológicos, tipo de aducto etc.) e interno (grado de transferencia de la L1, factores cognitivos, diferencias individuales como creencias, afecto, edad, aptitud, estilo de aprendizaje, motivación, personalidad). El propio status quo refleja a la perfección la heterogeneidad también de la investigación actual: algunos estudiosos - los generativistas mantienen que el desarrollo tanto de la L1 como de la L2 está sujeto a unas restricciones de maduración neurofisiológica y que estos cambios biológicos reducen progresivamente la capacidad del adulto de aprender una segunda lengua (Lenneberg 1967, Scovel 2000). Es en este marco en que se discute la accesibilidad de los principios y parámetros de la Gramática Universal (GU) para el aprendiente adulto de una segunda lengua (White 1989), entendiéndose la GU como la dotación biológica que constituye el órgano mental del lenguaje. Otros, en cambio, argumentan que son principalmente los factores psicosociales, 
tales como actitud, motivación o distancia cultural (el grado de asimilación de la cultura meta) los que determinan el éxito o el fracaso (Schumann 1978, Andersen 1983), aunque hoy se asume que la incidencia de los factores sociales es más bien secundaria (cfr. Ellis 1994:31).

Hagamos abstracción de todos estos factores para centrarnos en las propuestas generativistas que se mantienen principalmente sobre tres bases: la biológica, la psicológica y la gramatical. Primero, la base biológica: la capacidad lingüística entendida como un órgano mental que "crece" mediante la exposición al entorno es un interesante punto de partida para el estudio de la adquisición lingüística secundaria (L2) como una versión "especial", cuasi "patológica" (Liceras 1997:67), de la adquisición primaria (L1); en segundo lugar, su base psicológica - la visión modular de la mente y, por consiguiente, de la capacidad lingüística permite tratar tanto las representaciones gramaticales que realmente se adquieren como los mecanismos de aprendizaje y procesamiento que explican el proceso de adquisición; tercero, su avanzado modelo gramatical, cuyos rasgos principales son la universalidad y la variación paramétrica, posee los medios necesarios para una comparación de todas las lenguas, incluso las no-nativas (L2). (cfr. Liceras (1997).

La cuestión fundamental para los investigadores adscritos a este paradigma es la accesibilidad de la GU y sus parámetros en la edad adulta. Y, si la respuesta fuera afirmativa ¿se pueden cambiar sus valores una vez fijados? ¿O es que, por el contrario, la GU ha dejado de servir y son otros módulos cognitivos que adoptan la función del aprendizaje, como pueden ser los procesos generales de aprendizaje y el acceso a la retroalimentación negativa (negative feedback)?

Siguiendo a Liceras (1992:24-25) resumimos las tres posturas existentes:

- Inexistencia de acceso a la GU. En el conocimiento de la L2 intervienen otras facultades distintas a la GU, como los mecanismos de resolución de problemas o de aprendizaje no lingüístico. (Clahsen y Muysken (1986) Clahsen (1990, 1994), Schachter (1988) y Meisel (1997))

- Acceso directo a la GU. Eliminando el problema del periodo crítico, los parámetros de la L2 se fijan de la misma manera que los de la L1, a la que no se recurre en absoluto. Esta hipótesis tiene escasa viabilidad para el aprendizaje de una L2 por adultos.

- Acceso indirecto a la GU. La L1 tiene una función de intermediador entre la GU y la L2. La L1 se utiliza como hipótesis inicial con la que acercarse a la L2. Luego, el aprendiente es capaz de volver a fijar los parámetros en otro sentido.

Esta última opción es la más aceptada. Con ella quedarían explicadas sobre todo dos características del aprendizaje de una L2:

- las secuencias evolutivas, pues los aprendientes pueden adquirir antes las reglas que reflejen principios universales (no marcadas) y después las que no (marcadas). 
- el conocimiento de los universales lingüísticos puede estar relacionado, como apuntamos antes, con la transferencia: los aprendientes pueden transferir antes los rasgos de la $\mathrm{L} 1$ que reflejen principios de la GU que los que le sean ajenos.

Esta última característica no debe confundirse con un modelo de la adquisición basado en la transferencia. Liceras (1992) señala que "el modelo de acceso indirecto a la GU implica que la L1 es la primera realización de la GU, aun vigente en el cerebro, que permite el acceso a las opciones de los parámetros que no se dan en esa L1, aun cuando el aprendiz utilice las opciones de la L1 en las primeras etapas de la IL". En otras palabras, aunque el modelo paramétrico de adquisición aplicado a la L2 no se base en la transferencia, sí que reconoce que la L1, como capacidad cognitiva que es, juega un papel fundamental en la adquisición de la L2. El rechazo indiscriminado de la influencia de la L1, que se explicaba por la necesidad de separarse de las teorías conductistas del aprendizaje que habían hecho de la interferencia la clave para explicar la IL, ya no se siente como una necesidad. Por tanto, "la L1 vuelve a ocupar un papel central en la teoría de la adquisición de la L2." (Liceras 1992:25, el subrayado es mío).

\section{LA GU Y LA ADQUISICIÓN DEL OP ALEMÁN}

Por la visibilidad de las posiciones gramaticalizadas del verbo, el OP alemán ofrece un objeto de estudio muy apropiado para investigar la vigencia de la GU. Repasemos tres de las propuestas fundamentales en la bibliografía actual: Schwartz y Sprouse $(1994,1996)$, Vainikka y Young Scholten $(1994,1996)$ y Beck (1998). Los dos primeros asumen la plena accesibilidad de la GU, mientras que Beck lo ve restringido.

La discusión se remonta hasta el trabajo fundacional de Clahsen, Meisel y Pienemann (ZISA 1981) acerca de la adquisición del alemán por trabajadores italianos y españoles. Estas investigaciones mostraban que los aprendientes pasan por un primer estadio en que colocan el verbo en posición de núcleo inicial (SVO), contraria al orden del alemán adulto (SOV) y también a las fases de adquisición del alemán como L1 (60-70\% V-final). El debate gira en torno al origen de este sistema IL: influencia de la L1 - siempre a través de la GU- o la explicación funcionalista mediante una "estrategia canónica del orden de palabras" (Clahsen 1986)

\section{a. Vainikka y Young Scholten (1994): Influencia Parcial de la L1}

Vainikka y Young-Scholten (1994) investigan la adquisición (no-guiada) del alemán por hablantes nativos del turco y del coreano, siendo ambas lenguas nítidamente de núcleo final. De ser cierta la asunción de Clahsen y Muysken (1986), los sujetos coreanos y turcos también deberían producir las secuencias VO (orden canónico) desde el principio. Si, por el contrario, su IL mostraba los rasgos V-final de sus respectivas L1, la hipótesis de Clahsen se vería invalidada.

Las fases de maduración gramatical observadas fueron las siguientes: 


\begin{tabular}{|l|c|c|c|}
\hline & Movimiento verbal & Sujeto cero & Concordancia \\
\hline a. $(\mathrm{S}) \mathrm{OV}^{\text {-fin }}$ & no & sí & no \\
\hline b. $(\mathrm{S}) \mathrm{V}^{+\mathrm{fin}} \mathrm{O}\left(\mathrm{V}^{\text {-fin }}\right)$ & opcional & sí & no \\
\hline c. $\mathrm{SV}^{+\mathrm{fin}} \mathrm{O}\left(\mathrm{V}^{\text {-fin }}\right)$ & sí & no & sí \\
\hline
\end{tabular}

Con ello, se invalida la hipótesis del orden canónico de Clahsen y Muysken, puesto que el orden inicial de los sujetos es claramente el de su L1: OV. Pero comentemos las fases:

a. SV escueto:En la primera fase de "minimal trees"7, la transferencia se limita únicamente a las proyecciones léxicas (aquí SV) especificadas en cuanto a la posición del núcleo ("headedness" en la teoría X'). Las proyecciones funcionales FLEX y COMP no se encuentran todavía, ya que no se realizan fonéticamente. Van emergiendo en las siguientes fases, manifestándose en un aumento de las formas flexionadas y la realización fonética de los pronombres sujetos:

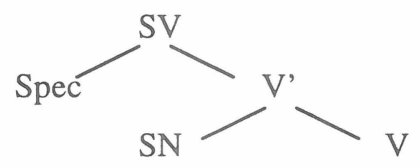

b. Proyección funcional aun no especificada Surge una proyección funcional "infradeterminada", cuyo núcleo sirve de lugar de destino para el movimiento verbal que todavía es opcional mientras que aun no es posible la concordancia sujeto verbo.<smiles>FP([Sn])[Pb][Sn]</smiles>

c. Sintagma FLEX. El sintagma funcional ya se ha consolidado plenamente. Los enunciados de los aprendientes ya se caracterizan por: concordancia sujeto-verbo, ascensión del verbo al segundo lugar, sujetos pronominales realizados. Por consiguiente, ya es posible la fijación del parámetro PRO-drop en el valor [-]. COMP aun no se manifiesta ya que apenas se producen oraciones incrustadas.

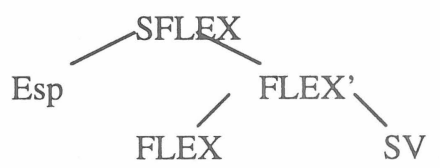

Sin embargo, las oraciones subordinadas (verbo final) no aparecen, a pesar de su existencia en ambas L1. Ello indica que en la adquisición de la L2 (alemán), a diferencia de la adqusición de la $\mathrm{L}^{8}$, los sintagmas funcionales SFLEX y SCOMP son ambos de núcleo

\footnotetext{
${ }^{7}$ Se establecen el menor número de proyecciones y posiciones posibles en cada fase evolutiva, o sea, sólo las estrictamente necesarias.

${ }^{8}$ cfr. tb. Clahsen 1982, Weissenborn 1994.
} 
inicial. A diferencia del sintagma verbal transferido en la fase 1, ahora, con los sintagmas funcionales, no opera la transferencia desde la L1.

\section{b. Schwartz y Sprouse $(1994,1996)$ : Pleno Acceso a la GU con Plena Transferencia de la L1.}

Con respecto a la presencia de las categorías funcionales en la IL temprana, Schwartz y Sprouse $(1994,1996)$ dan otra explicación contraria. Según deducen de su corpus 9 , procedente de la adquisición del OP en un aprendiente turco del alemán, la influencia de la L1 es tan absoluta que también suministra las categorías funcionales de la GU en la IL. La representación del "estado inicial" de turcos y coreanos ya consta de árboles estructurales altamente complejos. Como dijimos, en las dos lenguas, también las proyecciones funcionales COMP y FLEX son de núcleo final.

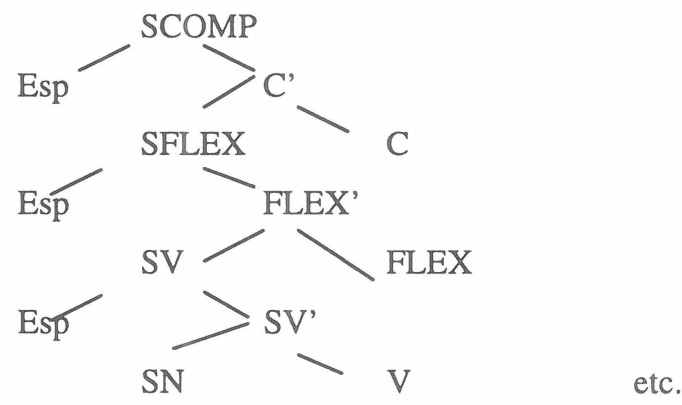

Ej. turco: "Me Hasan qué comió pregunté”, Me pregunté qué comería Hasan

Esta sería la representación de la fase (a), plenamente transferida de la L1. El hecho de que los aprendientes turcos no produzcan oraciones subordinadas con verbo final, tal como cabría esperar por la existencia de esta linearización su L1, no es debido, como afirman Vainikka y Young Scholten (1994), a la ausencia de COMP, sino a un proceso gradual de "acomodación al input "en cuya fase inicial el verbo es movido desde $\mathrm{V}^{\circ}$ (V-fin, L1) a $\mathrm{C}^{\circ}$ (V2, L2 en oraciones no subordinadas). El sujeto, a su vez, se mueve desde EspFLEX a EspSCOMP, movimiento prescrito por el filtro de caso nominativo de la GU.

El que los aprendientes no flexionen el verbo no significa que no sea finito (ya distinguen finito/no-finito en su L1). Sólo es que aun no dominan el paradigma morfológico de modo que lo que parece un inifinitivo en (a.) en realidad sólo es una forma finita no conforme con la lengua meta. La morfofonología ha de ser aprendida, lo mismo que en la adquisición de la L1. Schwartz hipotetiza que las categorías están ahí, como en la L1, pero aun no están léxicamente ocupadas o fonéticamente realizadas. La inexistencia fonética de un rasgo según Schwartz no es prueba suficiente de su ausencia absoluta.

\footnotetext{
${ }^{9}$ Procedente del proyecto de la Fundación de las Ciencias Europea (1988) Second Language Acquisition by Adult Immigrants. A Additional Activity of The European Science Foundation. Final Report, Estrasburgo, Nimega. Resulta muy interesante comparar las conclusiones formales de Schwartz (1994) con las funcionales de Klein / Perdue (1992) sobre el mismo corpus.
} 
Schwartz sigue con su argumentación a favor de su "Hipótesis de Influencia Absoluta" (y en contra de la "Hipótesis de Influencia Parcial", tb. "Hipótesis de las estructuras mínimas"), o sea, defendiendo la disponibilidad de todas las categorías, léxicas y funcionales, desde el comienzo mismo de la adquisición de la L2: el conocimiento de las categorías funcionales o flexivas (COMP, FLEX) puede estar ya determinado en la entrada léxica de los verbos como oir o querer. Estos verbos transitivos seleccionan como argumentos o un nombre (Quiero un helado) o una proposicion con categorías funcionales(Quiero [SFLEX comerme un helado] o Quiero [SCOMP que te comas el helado]). En este último caso, las categorías necesarias para la estructura oracional, COMP y FLEX, vendrían ya proyectadas desde el léxico como proyecciones funcionales.

Schwartz encuentra otro argumento a favor de la existencia inicial de las categorías funcionales de la GU por transferencia: son notorias las dificultades de los hablantes L1 francés para la colocación preverbal de los adverbios en L2 inglés (*'He travels often'). Teniendo una estructura-p común, su explicación estaría en la transferencia del parámetro de movimiento verbal al inglés (en francés, el verbo se mueve desde VP a FLEX).

Para resumir brevemente la "Hipótesis de la Influencia Absoluta" de Schwartz y Sprouse (1994): en el aprendiente no van madurando paulatinamente las estructuras funcionales necesarias (Vainikka y Young-Scholten 1994), sino que las distintas posiciones funcionales ya existentes se van llenando poco a poco, acomodándose al aducto. En este proceso se van cambiando los valores paramétricos de la secuenciación en los sintagmas funcionales COMP y FLEX.

\section{c. Beck (1998): The Locall Impairment Hypothesis.}

A diferencia de los autores anteriores, Beck, en un estudio experimental con sujetos de L1 inglés y L2 alemán, defiende que el acceso a la GU en la primera fase de adquisición es sólo parcial. El parámetro utilizado en su estudio es el del ascenso verbal obligatorio: en inglés (español, turco, coreano), el verbo permanece en su posición generada en la base, mientras que en alemán asciende a V2 desde V-final. Las predicciones respectivas de los estudios anteriores son:

a. Vainikka y Young Scholten $(1994,1996)$ :

- Estado inicial: no hay ascenso

- $\quad 1^{\mathrm{a}} \mathrm{s}$ fases: ascenso opcional

- Más tarde (cuando rasgo fuerza fijado en COMP): ascenso obligatorio

b. Schwartz y Sprouse $(1994,1996)$

- $\quad 1^{\text {a }}$ s fases: no hay ascenso (no hay cats. funcionales)

- Más tarde: ascenso obligatorio

Beck mantiene en su "perspectiva de la inhibición local" (Local Impairment View) que el movimiento de núcleo a núcleo (V2) pueda ser dificultado porque los aprendientes no tienen acceso a la parte del conjunto de rasgos bajo FLEX que les indica si la flexión es 
'fuerte' o 'debil'. En términos del "Programa Minimalista" son precisamente éstos los rasgos que exigen o prohiben el ascenso del verbo:

- francés (fuerte) ascenso obligatorio:

Il viens souvent a la ville

- inglés (débil) ascenso imposible

He often travels to Belgium

Beck encuentra evidencia en el breve tiempo de respuesta de sus sujetos ingleses a oraciones alemanas que no ascienden el verbo de $\mathrm{V}^{\circ}$ a $\mathrm{FLEX}^{\circ}$ a pesar de poseer esta el rasgo [+ fuerte], lo cual resulta en oraciones agramaticales como (10):

*Dann der Vater liest die Zeitung

Explica los datos asumiendo

- que los aprendientes principiantes no poseen categorías funcionales, sólo SV, y por tanto no pueden ascender el verbo

- que los aprendientes más avanzados tienen acceso restringido al rasgo 'fuerza' de FLEX y por lo tanto sólo permiten ascenso opccional cuando es obligatorio.

\section{CONCLUSIÓN}

La investigación de los procesos de adquisición de una segunda lengua en el marco generativista ha alcanzado una gran complejidad teórica y experimental. Sus resultados, sin duda, siguen dependiendo de los supuestos teóricos en los que se basan. El más importante de estos supuestos es la vigencia, parcial o absoluta, de la GU en edad adulta a través de los parámetros de la primera lengua.

Después de todo, creo que Mark Twain, de haber leido a Chomsky, podría haber encontrado algo de lógica en esa "terrible lengua alemana". O también le podría haber dado por redactar otra glosa titulada "la terrible gramática generativa".

\section{BIBLIOGRAFÍA}

Andersen, R.(ed.) (1981), New Dimensions in Second Language Acquisition Research, Newbury House, Rowley Mass.

Bach, E. (1962), "The Order of Elements in a Transformational Grammar of German", Language, 38, 263-9.

Beck, M.L. (1998), "L2 acquisition and obligatory head movement”, en Studies of Second Language Acquisition, 20, 311-348.

Bierwisch, M. (1963), Grammatik des deutschen Verbs, Studia Grammatika 2, Akademieverlag, Berlin. 
Chomsky, N. (1965), Aspects of the Theory of Syntax, MIT Press, Cambridge, Mass. (Trad. esp.Aspectos de la teoría de la sintaxis, Aguilar, Madrid)

(1981), Lectures on Government and Binding, MIT Press, Cambridge (Massachussets)

(1982), Some Concepts and Consequences of the Theory of Government and Binding, MIT Press, Cambridge; trad.cast, La nueva sintaxis. Teoría de la rección y el ligamiento, Paidós, Barcelona.

(1993), “A Minimalist Program for Linguistic Theory”, en Hale, K, y Keyser, S.J.(eds.)(1993), The View from Building 20. Essays in Linguistics in Honor of Sylvain Bromberger, MIT Press, Cambridge (MA).

Clahsen, H. (1990), "Constraints on parameter setting: A grammatical analysis of some acquisition stages in German child language", Language Acquisition1 (4), 361-391.

- y Muysken, P.(1986), "The availability of Universal Grammar to adult and child learners - the study of the acquisition of German word order", Second Language Research 2, pp.93-119

Comrie, B. (1981), Language Universals and Linguistic Typology. Syntax and Morphology, Blackwell, Oxford, (1988, vers.esp.), Universales del lenguaje y tipología lingüística, Gredos, Madrid.

Demonte,V.(1989), Teoría sintáctica. De las estructuras a la rección, Síntesis, Madrid

Ellis, R. (1994), The Study of Second Language Acquisition, OUP, Oxford.

Greenberg,. J.H. (ed.)(1963), Universals of Language, M.I.T. Press, Cambrigde, Mass.

Haider, H. y PRINZHORN, M. (eds.)(1986), Verb second Phenomena in Germanic Languages, Foris, Dordrecht.

Hernanz, M.L. y Brucart, J.M. (1987), La sintaxis, Editorial Crítica, Barcelona.

Ingram, D. y Thompson, W. (1996), "Early syntactic acquisition in German: Evidence for the Modal Hypothesis, Language, 72, 97-120.

Kayne, R. (1994), The Antisymmetry of Syntax, MIT Press, Cambridge MA,

Klein, W. y Perdue, C. (1992), Utterance Structure, Benjamins, Amsterdam

Lasnik, H. y Saito, M. (1984), "On the nature of proper government", Linguistic Inquiry, 15, 2, 235-289.

Lenneberg, E. (1967), Biological Foundations of Language, Wiley and Sons, Nueva York. (Trad.esp. (1975) Fundamentos biológicos del lenguaje, Alianza, Madrid)

Liceras Muñoz, J. (1992), La adquisición de las lenguas extranjeras, Visor, Madrid.

- (1997), "The Now and Then of L2 Growing Pains", en Díaz, L. y Pérez, C. (coords.)(1997), Actas de EUROSLA 2, Pompeu Fabra, Barcelona.

Meisel, J.M. (1997), "The acquisition of syntax of negation in French and German": Contrasting first and second language development", Second Language Research, 13, 227-263. 
Platzack, C. (1983), "Germanic Word Order and the COMP/INFL Parameter", en Working Papers in Scandinavian Syntax 2. Linguistics Department, University of Trondheim.

Poeppel, D. y Wexler, K. (1993), "The Full Competence Hypothesis of Clause Structure in Early German”, Language, 69, 1-33.

Schumann, J.H.(1978), The pidginization process: A model for second language acquisition, Newbury House, Rowley Mass.

Schwartz, B.D. y Sprouse, R.A. (1994), "Word order and nominative case in non-native language acquisition. A longitudinal study of (L1 Turkish) German Interlanguage”, en Hoekstra,T. y Schwartz, B.D., Language acquisition studies in generative grammar, Benjamins, Amsterdam.

- (1996), "L2 cognitive states and the Full Transfer/Full Access Model" [edición especial], Second Language Research, 12, 40-72.

Scovel, T. (2000) "A Critical Review of the Critical Period", ARAL 20, 213-224.

Siewierska, A. (1994), "Word Order and Linearization", en Asher, R.E.(ed.), The Encyclopedia of Language and Languages, p.4993- 4999, Pergamon, Oxford

Thiersch, C. (1978), Topics in German Syntax, MIT-Dissertation, Cambridge, Mass.

Travis, L. (1991), "Parameters of Phrase Structure and Verb-Second Phenomena", en Freidin, R. (ed.), (1991), Principles and Parameters in Comparative Grammar, MIT Press, Cambridge,Mass.

Twain,M. ( $\left.{ }^{1} 1880,1985\right)$, Bummel durch Europa, Insel, FrankfurtM., título del original: A tramp abroad.

Vainikka, A. y Young-Scholten, M. (1994), "Direct Access to X-bar Theory. Evidence from Corean and Turkish adults learning German", en Hoekstra, T. y Schwartz, B.D. (eds.), Language acquisition studies in generative grammar, (pp.265-316), Benjamins, Amsterdam.

- (1996), "Gradual development of L2 phrase structure" [edición especial] Second Language Research, 12, 7-39.

Weissenborn, J. (1994), "Constraining the child's grammar. Local well formedness in the development of the verb movement in German and French", en Lust, B., Sunyer, M., Whitman, J. (eds.), Heads, Projections and Learnability, Lawrence Erlbaum, Hillsdale, Nueva Jersey.

White, L.(1989), Universal Grammar and Second Language Acquisition, Benjamin, Amsterdam.

Zwart, J.-W.(1991), "Clitics in Dutch: Evidence for the position of Infl.", Groninger Arbeiten zur Germanistischen Linguistik, 33, 71-92. 